\title{
ENTROPY FOR GROUP ENDOMORPHISMS AND HOMOGENEOUS SPACES
}

\author{
BY \\ RUFUS BOWEN
}

\begin{abstract}
Topological entropy $h_{d}(T)$ is defined for a uniformly continuous map on a metric space. General statements are proved about this entropy, and it is calculated for affine maps of Lie groups and certain homogeneous spaces. We compare $h_{d}(T)$ with measure theoretic entropy $h(T)$; in particular $h(T)=h_{d}(T)$ for Haar measure and affine maps $T$ on compact metrizable groups. A particular case of this yields the wellknown formula for $h(T)$ when $T$ is a toral automorphism.
\end{abstract}

Introduction. We shall study topological entropy, concentrating on its relation to measure theoretic entropy and algebraic examples. Our topological entropy $h_{d}(T)$ is defined (in §2) for a uniformly continuous map $T$ on a metric space $(X, d)$. In [1] a topological entropy $h(T)$ was defined for a continuous map on a compact topological space; if the space is compact metric then $h(T)=h_{d}(T)$. An essential part of this paper is the computation of $h_{d}(T)$ for certain maps on noncompact spaces.

Suppose $\mu$ is a Borel measure on $\mu(X)=1$, and $\mu$ is $T$-invariant (i.e. $\mu\left(T^{-1}(A)\right.$ ) $=\mu(A)$ for every Borel set $A)$. One can then define a measure theoretic entropy $h_{\mu}(T)$ as follows: Call $\alpha=\left\{A_{1}, \ldots, A_{r}\right\}$ a (finite) measurable partition of $X$ if the $A_{i}$ are disjoint measurable subsets of $X$ covering $X$. Now set

$$
H_{m}(\alpha)=\sum_{1 \leqq i_{0}, \ldots, i_{m-1} \leqq r}-\mu\left(\bigcap_{k=0}^{m-1} T^{-k} A_{i_{k}}\right) \log \mu\left(\bigcap_{k=0}^{m-1} T^{-k} A_{i_{k}}\right) .
$$

Then the limit $h_{\mu}(T, \alpha)=\lim _{m \rightarrow \infty}(1 / m) H_{m}(\alpha)$ exists and one defines

$$
h_{\mu}(T)=\sup \left\{h_{\mu}(T, \alpha): \alpha \text { is a finite measurable partition of } X\right\} \text {. }
$$

(See [6] for details about measure theoretic entropy.)

Two points in $X$ are separated by $\alpha=\left\{A_{1}, \ldots, A_{r}\right\}$ provided they lie in different $A_{i}$ 's. We shall use the following fact to compute entropy:

Fact (see [6]). Let $\left\{\alpha_{k}\right\}_{k=0}^{\infty}$ be a sequence of measurable partitions of $X$ satisfying the following property: If $x, y \in X$ are distinct there is an $n(x, y)$ such that $\alpha_{k}$ separates $x$ and $y$ whenever $k \geqq n(x, y)$. Then $h_{\mu}(f)=\sup _{k} h\left(T, \alpha_{k}\right)$.

As is generally known, if $T: G \rightarrow G$ is a surjective endomorphism of a compact metrizable group, then $T$ preserves Haar measure $\mu$. For such a $T$ we show that the

Received by the editors October 17, 1969 and, in revised form, February 2, 1970.

AMS 1969 subject classifications. Primary 2870, 2875; Secondary 5482.

Key words and phrases. Entropy, $T$-homogeneous measure.

Copyright (C) 1971, American Mathematical Society 
two entropies we have are equal (Theorem 9): $h_{\mu}(T)=h_{d}(T)$. For the case where $G$ is a Lie group we show that $T$ and its derivative at the identity have the same topological entropy (Corollary 11). We then calculate the topological entropy of a linear map in terms of its eigenvalues (Theorem 15). This series of results yields the formula for the entropy of an endomorphism (in fact of any affine map) of a Lie group, a formula already well known for tori (see [7], [8], [9], [10] and [12]). Finally we prove the formula for an affine map of a homogeneous space $G / \Gamma$ where $\Gamma$ is a uniform discrete subgroup of $G$ (Theorem 20 and the remark following it).

Other results of some interest are noted along the way. This paper benefited considerably from conversations with C. Bones, W. Parry and P. Walters.

1. Topological entropy. Let $(X, d)$ be a metric space and $T: X \rightarrow X$ be uniformly continuous (abbreviated $T \in \mathrm{UC}(X, d)$ ). A set $E \subset X$ is $(n, \varepsilon)$-separated if for any distinct $x, y \in E$ there is a $j$ such that $0 \leqq j<n$ and $d\left(T^{j}(x), T^{j}(y)\right)>\varepsilon$. A set $F(n, \varepsilon)$-spans another set $K$ (with respect to $T$ ) provided that for each $x \in K$ there is a $y \in F$ for which $d\left(T^{j}(x), T^{j}(y)\right) \leqq \varepsilon$ for all $0 \leqq j<n$.

For a compact set $K \subset X$ let $r_{n}(\varepsilon, K)$ be the smallest cardinality of any set $F$ which $(n, \varepsilon)$-spans $K$ (with respect to $T$ ) and let $s_{n}(\varepsilon, K)$ denote the largest cardinality of any $(n, \varepsilon)$-separated set $E$ contained in $K$. We write $r_{n}(\varepsilon, K, T)$ or $s_{n}(\varepsilon, K, T)$ if we wish to stress the dependence on $T$. Finally define

and

$$
\bar{r}_{T}(\varepsilon, K)=\limsup _{n \rightarrow \infty} \frac{1}{n} \log r_{n}(\varepsilon, K)
$$

$$
\bar{s}_{T}(\varepsilon, K)=\limsup _{n \rightarrow \infty} \frac{1}{n} \log s_{n}(\varepsilon, K) .
$$

LEMMA 1. (i) $r_{n}(\varepsilon, K) \leqq s_{n}(\varepsilon, K) \leqq r_{n}\left(\frac{1}{2} \varepsilon, K\right)<\infty$.

(ii) For $\varepsilon_{1}<\varepsilon_{2}, \bar{r}_{T}\left(\varepsilon_{1}, K\right) \geqq \bar{r}_{T}\left(\varepsilon_{2}, K\right)$ and $\bar{s}_{T}\left(\varepsilon_{1}, K\right) \geqq \bar{s}_{T}\left(\varepsilon_{2}, K\right)$.

Proof. A maximal $(n, \varepsilon)$-separated subset of $K(n, \varepsilon)$-spans $K$; so $r_{n}(\varepsilon, K) \leqq s_{n}(\varepsilon, K)$. Suppose $E \subset K$ is $(n, \varepsilon)$-separated and $F\left(n, \frac{1}{2} \varepsilon\right)$-spans $K$; by the definition of spanning, for each $x \in K$ we can pick a $g(x) \in F$ such that $d\left(T^{j}(x), T^{j}(g(x))\right) \leqq \frac{1}{2} \varepsilon$ for all $0 \leqq j<n$. If $g(x)=g(y)$, then $d\left(T^{j}(x), T^{j}(y)\right) \leqq \varepsilon$ for all $0 \leqq j<n$; since $E$ is $(n, \varepsilon)$ separated, it follows that $g$ is injective on $E$. Hence card $F \geqq \operatorname{card} E$ and $r_{n}\left(\frac{1}{2} \varepsilon, K\right)$ $\geqq s_{n}(\varepsilon, K)$. As $T \in \mathrm{UC}(X, d)$ there is a $\delta>0$ such that $d\left(T^{j}(x), T^{j}(y)\right)<\frac{1}{2} \varepsilon$ for all $0 \leqq j<n$ whenever $d(x, y)<\delta$; then $r_{n}\left(\frac{1}{2} \varepsilon, K\right)$ is at most the number of $\delta$-balls needed to cover $K$ (finite as $K$ is compact).

(ii) is trivial.

By the lemma the following definition makes sense:

Definition 2. For $T \in \mathrm{UC}(X, d)$ and $K \subset X$ compact, set

and

$$
h_{d}(T, K)=\lim _{\varepsilon \rightarrow 0} \bar{r}_{T, d}(\varepsilon, K)=\lim _{\varepsilon \rightarrow 0} \bar{s}_{T, d}(\varepsilon, K)
$$

$$
h_{d}(T)=\sup _{K \text { compact }} h_{d}(T, K) .
$$


REMARK. In the above definition we stress the dependence on the metric $d$ used. Notice that $h_{d}(T, K) \leqq \max _{i} h_{d}\left(T, K_{i}\right)$ if $K \subset K_{1} \cup \cdots \cup K_{m}$. If $X$ is compact, then $h_{d}(T)=h_{d}(T, X)$. Also, in computing $h_{d}(T)$, one need only consider $K$ with small diameter.

Metrics $d$ and $d^{\prime}$ on $X$ are uniformly equivalent if $\operatorname{id}_{X}:(X, d) \rightarrow\left(X, d^{\prime}\right)$ and $\mathrm{id}_{X}:\left(X, d^{\prime}\right) \rightarrow(X, d)$ are uniformly continuous maps of metric spaces. In this case $T \subset \mathrm{UC}(X, d)$ if and only if $T \in \mathrm{UC}\left(X, d^{\prime}\right)$.

Proposition 3. If d and d' are uniformly equivalent metrics on $X$ and $T \in \mathrm{UC}(X, d)$, then $h_{d}(T)=h_{d^{\prime}}(T)$.

Proof. Given $\varepsilon_{1}>0$, choose $\varepsilon_{2} \geqq \varepsilon_{3}>0$ such that $d(x, y) \leqq \varepsilon_{1}$ whenever $d^{\prime}(x, y) \leqq \varepsilon_{2}$, and $d^{\prime}(x, y) \leqq \varepsilon_{2}$ whenever $d(x, y) \leqq \varepsilon_{3}$. Let $K \subset X$ be compact. Then an $\left(n, \varepsilon_{2}\right)-$ spanning set for $K$ with respect to $d^{\prime}$ is an $\left(n, \varepsilon_{1}\right)$-spanning set for $K$ with respect to $d$. Hence

$$
r_{n}\left(\varepsilon_{1}, K, d\right) \leqq r_{n}\left(\varepsilon_{2}, K, d^{\prime}\right)
$$

Similarly

$$
r_{n}\left(\varepsilon_{2}, K, d^{\prime}\right) \leqq r_{n}\left(\varepsilon_{3}, K, d\right)
$$

Letting $n \rightarrow \infty$,

$$
\bar{r}_{T, d}\left(\varepsilon_{1}, K\right) \leqq \bar{r}_{T, d}\left(\varepsilon_{2}, K\right) \leqq \bar{r}_{T, d}\left(\varepsilon_{3}, K\right)
$$

Letting $\varepsilon_{1} \rightarrow 0$,

$$
h_{d}(T, K)=h_{d} \cdot(T, K) .
$$

REMARK. When $X$ is compact, all equivalent metrics on $X$ are uniformly equivalent; also any continuous $T: X \rightarrow X$ is uniformly continuous. The common value in this case of all the $h_{d}(T)$ 's is just the topological entropy defined in [1] (for a proof, see [2]). For $T: R^{n} \rightarrow R^{n}$ a linear map, we shall always take the metric $d$ to be one arising from a norm; $h_{d}(T)$ is then independent of the norm used.

Proposition 4. (i) $h_{d}\left(T^{m}\right)=m h_{d}(T)$ for any $T \in \mathrm{UC}(X, d)$ and any $m>0$.

(ii) Suppose $T_{1} \in \mathrm{UC}\left(X_{1}, d_{1}\right)$ and $T_{2} \in \mathrm{UC}\left(X_{2}, d_{2}\right)$. Define $d$ on $X_{1} \times X_{2}$ by $d\left(\left(x_{1}, x_{2}\right),\left(y_{1}, y_{2}\right)\right)=\max \left\{d_{1}\left(x_{1}, y_{1}\right), d_{2}\left(x_{2}, y_{2}\right)\right\}$. Then $h_{d}\left(T_{1} \times T_{2}\right)=h_{d_{1}}\left(T_{1}\right)+h_{d_{2}}\left(T_{2}\right)$.

REMARK. For $X$ compact, [1] has a proof of (i) and [4] of (ii).

Proof. (i) Clearly $r_{n}\left(\varepsilon, K, T^{m}\right) \leqq r_{m n}(\varepsilon, K, T)$; it follows that $h_{d}\left(T^{m}\right) \leqq m h_{d}(T)$. Given $\varepsilon>0$, choose $\delta>0$ (since $T \in \mathrm{UC}(X, d)$ ) such that $d\left(T^{j}(x), T^{j}(y)\right) \leqq \varepsilon$ for all $0 \leqq j<m$ whenever $d(x, y) \leqq \delta$. One sees that an $(n, \delta)$-spanning set for $K$ with respect to $T^{m}$ is automatically an $(m n, \varepsilon)$-spanning set for $K$ with respect to $T$. Hence $r_{m n}(\varepsilon, K, T) \leqq r_{n}\left(\delta, K, T^{m}\right)$; we get $m h_{d}(T) \leqq h_{d}\left(T^{m}\right)$.

(ii) If $E_{i}(i=1,2)$ is an $(n, \varepsilon)$-spanning set for $K_{i}$ (compact subset of $X_{i}$ ), then $E_{1} \times E_{2}$ is an $(n, \varepsilon)$-spanning set for $K_{1} \times K_{2}$ with respect to $T_{1} \times T_{2}$. Thus $r_{n}\left(\varepsilon, K_{1} \times K_{2}, T_{1} \times T_{2}\right) \leqq r_{n}\left(\varepsilon, K_{1}, T_{1}\right)+r_{n}\left(\varepsilon, K_{2}, T_{2}\right)$ and so $h_{d}\left(T_{1} \times T_{2}, K_{1} \times K_{2}\right) \leqq$ $h_{d_{1}}\left(T_{1}, K_{1}\right)+h_{d_{2}}\left(T_{2}, K_{2}\right)$. 
If $F_{i} \subset K_{i}$ is an $(n, \varepsilon)$-separated set with respect to $T_{i}$, then $F_{1} \times F_{2} \subset K_{1} \times K_{2}$ is $(n, \varepsilon)$-separated with respect to $T_{1} \times T_{2}$. Hence $s_{n}\left(\varepsilon, K_{1} \times K_{2}, T_{1} \times T_{2}\right) \geqq s_{n}\left(\varepsilon, K_{1}, T_{1}\right)$ $+s_{n}\left(\varepsilon, K_{2}, T_{2}\right)$ and $h_{d}\left(T_{1} \times T_{2}, K_{1} \times K_{2}\right) \geqq h_{d_{1}}\left(T_{1}, K_{1}\right)+h_{d_{2}}\left(T_{2}, K_{2}\right)$.

Let $\pi_{i}: X_{1} \times X_{2} \rightarrow X_{i}$ be the projection map. If $K \subset X_{1} \times X_{2}$ is compact, then $K_{1}=\pi_{1}(K)$ and $K_{2}=\pi_{2}(K)$ are compact and $K \subset K_{1} \times K_{2}$. It follows that

$$
\begin{aligned}
h_{d}\left(T_{1} \times T_{2}\right) & =\sup _{K} h_{d}\left(T_{1} \times T_{2}, K\right)=\sup _{K_{1}, K_{2}} h_{d}\left(T_{1} \times T_{2}, K_{1} \times K_{2}\right) \\
& =\sup _{K_{1}} h_{d_{1}}\left(T_{1}, K_{1}\right)+\sup _{K_{2}} h_{d_{2}}\left(T_{2}, K_{2}\right)=h_{d_{1}}\left(T_{1}\right)+h_{d_{2}}\left(T_{2}\right) .
\end{aligned}
$$

2. Measures and groups. Standing hypothesis. For the rest of the paper $(X, d)$ will always be a locally compact metric space.

We shall now let measures enter the scene. The major result is due to $L$. Goodwyn [3].

THEOREM (GoOdWyn). Let $(X, d)$ be a compact metric space, $T: X \rightarrow X$ continuous and $\mu$ a T-invariant Borel measure on $X$ with $\mu(X)=1$. Then $h_{\mu}(T) \leqq h_{d}(T)$.

We shall give a proof which only works for $X$ finite dimensional; our paper will then be self-contained for such $X . X$ has covering dimension at most $m$ if every open cover $\mathscr{A}$ of $X$ has a refinement $\mathscr{B}$ such that each point of $X$ is in at most $m+1$ members of $\mathscr{B}$. An $m$-manifold satisfies this condition [5].

Proposition 5. Goodwyn's theorem is true for $X$ having covering dimension at most $m$.

Proof. Let $\mathscr{B}_{\varepsilon}=\left\{B_{1}, \ldots, B_{r}\right\}$ be an open cover of $M$ with diam $B_{i}$ less than $\varepsilon$ and each point of $X$ in at most $m+1$ members of $\mathscr{B}_{\varepsilon}$. Let $\alpha_{\varepsilon}=\left\{A_{1}, \ldots, A_{r}\right\}$ be a measurable partition of $X$ with $\bar{A}_{i} \subset B_{i}$. For each $x \in X$ choose an open neighborhood $C_{x}$ of $x$ intersecting at most $m+1 A_{i}$ 's (we can avoid $\bar{A}_{i}$ when $x \notin B_{i}$ ). Let $\mathscr{C}=\left\{C_{x_{1}}, \ldots, C_{x_{s}}\right\}$ cover $X$. Let $\delta>0$ be a Lebesgue number for $\mathscr{C}$, i.e. for each $y \in X$ there is a $C(y) \in \mathscr{C}$ such that $\bar{B}_{\delta}(y)=\{x \in X: d(x, y) \leqq \delta\} \subset C(y)$.

Let $S_{n}$ be an $(n, \delta)$-spanning set for $X$ with respect to $T$. Let $T_{n}=\left\{\left(i_{0}, \ldots, i_{n-1}\right)\right.$ : $y \in S_{n}$ such that $A_{i_{1}} \cap C\left(T^{j}(y)\right) \neq \varnothing$ for all $\left.0 \leqq j<n\right\}$. Now suppose $x \in A\left(i_{0}, \ldots, i_{n-1}\right)$ $\equiv \bigcap_{j=0}^{n-1} T^{-j} A_{i}$. Pick $y \in S_{n}$ so that $d\left(T^{j}(x), T^{j}(y)\right) \leqq \delta$ for all $0 \leqq j<n$. Then $T^{j}(x) \in A_{i}, \cap C\left(T^{j}(y)\right)$ and so $\left(i_{0}, \ldots, i_{n-1}\right) \in T_{n}$. Let $U_{n}=\left\{\left(i_{0}, \ldots, i_{n-1}\right)\right.$ : $\left.A\left(i_{0}, \ldots, i_{n-1}\right) \neq \varnothing\right\} ; U_{n} \subset T_{n}$. As $C\left(T^{j}(y)\right)$ can intersect at most $m+1 A_{k}$ 's,

Hence

$$
\text { card } T_{n} \leqq(m+1)^{n} \text { card } S_{n} \text {. }
$$

$$
\text { card } U_{n} \leqq \operatorname{card} T_{n} \leqq r_{n}(\delta, X, T)(m+1)^{n} .
$$

Now it is a basic fact that (see [6, p. 12] for example) that $H_{n}\left(\alpha_{\varepsilon}\right) \leqq \log$ card $U_{n}$. Hence

$$
\begin{aligned}
h_{\mu}\left(T, \alpha_{\varepsilon}\right) & =\lim _{n \rightarrow \infty} \frac{1}{n} H_{n}\left(\alpha_{\varepsilon}\right) \leqq \limsup _{n \rightarrow \infty} \frac{1}{n} \log (m+1)^{n} r_{n}(\delta, X) \\
& \leqq \log (m+1)+\bar{r}_{T}(\delta, X) \leqq \log (m+1)+h_{d}(T) .
\end{aligned}
$$


Letting $\varepsilon \rightarrow 0$, we get

$$
h_{\mu}(T)=\sup _{\varepsilon} h_{\mu}\left(T, \alpha_{\varepsilon}\right) \leqq \log (m+1)+h_{d}(T) .
$$

Applying this inequality to $T^{n}$ and using Proposition 4(i):

$$
h_{\mu}(T)=\frac{1}{n} h_{\mu}\left(T^{n}\right) \leqq \frac{1}{n} \log (m+1)+\frac{1}{n} h_{d}\left(T^{n}\right)=\frac{1}{n} \log (m+1)+h_{d}(T) .
$$

Letting $n \rightarrow \infty, h_{\mu}(T) \leqq h_{d}(T)$.

Definition 6. Suppose $T \in \mathrm{UC}(X, d)$. Set $D_{n}(x, \varepsilon, T)=\bigcap_{k=0}^{n-1} T^{-k} B_{\varepsilon}\left(T^{k}(x)\right)$ where $B_{\varepsilon}(y)=\{z \in X: d(z, y)<\varepsilon\}$. A Borel measure $\mu$ on $X$ is T-homogeneous provided that

(i) $\mu(K)<\infty$ for $K \subset X$ compact.

(ii) $\mu(K)>0$ for some compact $K$.

(iii) For each $\varepsilon>0$ there exist $\delta>0$ and $c>0$ such that

$$
\mu\left(D_{n}(y, \delta, T)\right) \leqq c \mu\left(D_{n}(x, \varepsilon, T)\right)
$$

for all $n \geqq 0$ and all $x, y \in X$.

For such a $\mu$ we define

$$
k(\mu, T)=\lim _{\varepsilon \rightarrow 0} \limsup _{n \rightarrow \infty}-\frac{1}{n} \log \mu\left(D_{n}(y, \varepsilon, T)\right) .
$$

By (iii) this does not depend on the $y$ used.

ReMARK. One easily sees that $\mu$ being $T$-homogeneous and $k(\mu, T)$ depend only on the uniform equivalence class of $d$.

Proposition 7. Suppose $T \in \mathrm{UC}(K, d)$ and $\mu$ is T-homogeneous. Then $h_{d}(T)$ $=k(\mu, T)$. If $X$ is compact, $\mu(X)=1$ and $\mu$ is T-invariant as well as T-homogeneous, then $h_{\mu}(T)=k(\mu, T)$.

Proof. Consider $K \subset X$ compact. Using (i) and local compactness find a neighborhood $U$ of $K$ with $\mu(U)<\infty$. Consider $\varepsilon>0$ so small that $B_{\varepsilon}(K) \subset U$. If $E \subset K$ is $(n, \varepsilon)$-separated, then one checks from the definitions that $\bigcup_{x \in E} D_{n}\left(x, \frac{1}{2} \varepsilon, T\right) \subset U$ is a disjoint union. Choose $\delta, c$ so that $\mu\left(D_{n}(y, \delta, T)\right) \leqq c \mu\left(D_{n}\left(x, \frac{1}{2} \varepsilon, T\right)\right)$ for all $x, y$. Then $\mu\left(D_{n}(y, \delta, T)\right) s_{n}(\varepsilon, K) \leqq c \mu(U)$ and so

$$
\bar{s}_{T}(\varepsilon, K) \leqq \limsup _{n \rightarrow \infty}-\frac{1}{n} \log \mu\left(D_{n}(y, \delta, T)\right) .
$$

Letting $\varepsilon \rightarrow 0, h_{d}(T, K) \leqq k(\mu, T)$. Hence $h_{d}(T) \leqq k(\mu, T)$.

Now consider $K$ with $\mu(K)>0$. If $F(n, \delta)$-spans $K$, then $\bigcup_{x \in F} D_{n}(x, 2 \delta, T) \supset K$. Given $\varepsilon>0$, choose $\delta>0$ and $c>0$ so that $\mu\left(D_{n}(x, 2 \delta, T)\right) \leqq c \mu\left(D_{n}(y, \varepsilon, T)\right)$ for all $x, y$ and all $n \geqq 0$. Then

$$
c \mu\left(D_{n}(y, \varepsilon, T)\right) r_{n}(\delta, K) \geqq \mu(K)>0
$$


and we get

$$
\bar{r}_{T}(\delta, K) \geqq \limsup _{n \rightarrow \infty}-\frac{1}{n} \log \mu\left(D_{n}(y, \varepsilon, T)\right) .
$$

Letting $\varepsilon \rightarrow 0, h_{d}(T, K) \geqq k(\mu, T)$. Hence $h_{d}(T)=k(\mu, T)$.

Now suppose $X$ is compact and the additional assumptions on $\mu$ of the second statement hold. By Goodwyn's theorem

$$
h_{\mu}(T) \leqq h_{d}(T)=k(\mu, T) .
$$

Let $\varepsilon>0$; choose $\delta$ and $c$ with $\mu\left(D_{n}(x, \delta, T)\right) \leqq c \mu\left(D_{n}(y, \varepsilon, T)\right)$ for all $x, y$ and all $n \geqq 0$. Let $\alpha=\left\{A_{1}, \ldots, A_{r}\right\}$ be a measurable partition of $X$ with diam $A_{i}<\delta$ for all $i$. If $x \in A\left(i_{0}, \ldots, i_{n-1}\right) \equiv \bigcap_{k=0}^{n-1} T^{-k} A_{i_{k}}$, then $A\left(i_{0}, \ldots, i_{n-1}\right) \subset D_{n}(x, \delta, T)$ and so $\mu\left(A\left(i_{0}, \ldots, i_{n-1}\right)\right) \leqq c \mu\left(D_{n}(y, \varepsilon, T)\right)$. It follows that (see the introduction)

$$
h_{\mu}(T) \geqq h_{\mu}(T, \alpha) \geqq \limsup _{n \rightarrow \infty}-\frac{1}{n} \log \mu\left(D_{n}(y, \varepsilon, T)\right) .
$$

Letting $\varepsilon \rightarrow 0, h_{\mu}(T) \geqq k(\mu, T)$.

EXAMPLE 8. Our prime example is $X=G$, a locally compact metrizable group. $G$ has a right invariant metric $d$ (see $[14$, p. 34]) and a right Haar measure $\mu$. For $g \in G$ define $R_{g}: G \rightarrow G$ by $R_{g}(x)=x g$. We shall consider $T$ of the form $T=R_{g} \circ A$ where $g \in G$ and $A$ is a continuous endomorphism of $G$. Such $T$ include endomorphisms $(g=e)$ and left translations $\left(A(x)=g x g^{-1}\right)$. One easily sees that such a $T$ is uniformly continuous.

$\mu$ is $T$-homogeneous and $k(\mu, T)=k(\mu, A)$.

Proof. It is enough to show that $D_{n}(x, \varepsilon, T)=D_{n}(e, \varepsilon, A) x$ since $\mu$ is a right Haar measure. We prove $T^{-k} B_{\varepsilon}\left(T^{k}(x)\right)=\left(A^{-k} B_{\varepsilon}(e)\right) x$ by induction. For $k=0$ this is obvious.

$$
\begin{aligned}
T^{-(k+1)} B_{\varepsilon}\left(T^{k+1}(x)\right) & =T^{-1} T^{-k} B_{\varepsilon}\left(T^{k}(T(x))\right)=T^{-1}\left[\left(A^{-k} B_{\varepsilon}(e)\right) T(x)\right] \\
& =A^{-1} \circ R_{g}-1\left[\left(A^{-k} B_{\varepsilon}(e)\right) A(x) g\right]=A^{-1}\left[\left(A^{-k} B_{\varepsilon}(e)\right) A(x)\right] \\
& =\left(A^{-(k+1)} B_{\varepsilon}(e)\right) x
\end{aligned}
$$

where we use in the last step the fact that, since $A$ is an endomorphism, $A^{-1}(C A(x))$ $=A^{-1}(C) x$ for any $C \subset G$. Intersecting over $0 \leqq k<n$ we have what we desired.

THEOREM 9. Suppose $G$ is a compact metrizable group, $A$ a surjective endomorphism, and $\mu$ normalized right Haar measure. Then

$$
h_{\mu}\left(R_{g} \circ A\right)=h_{\mu}(A)=k(\mu, T)=h_{d}(A) .
$$

Proof. In the above circumstance $\mu$ is $R_{g} \circ A$ - and $A$-invariant. Apply Proposition 7 to Example 8.

REMARK. $h_{\mu}(A)=h_{d}(A)$ was proved for tori by K. Berg [12] and suspected in general [1]. 
Proposition 10. Suppose $T: N \rightarrow N, S: M \rightarrow M$ and $\pi: N \rightarrow M=\pi(N)$ are differentiable maps of manifolds with $\pi \circ T=S \circ \pi$. Let $d$ and $d^{\prime}$ be metrics on $N$ and $M$ respectively. Suppose that $\mu$ is a (positive) smooth measure on $N$ which is $T$ homogeneous (with respect to $d$ ) and $\mu^{\prime}$ is a (positive) smooth measure which is $S$ homogeneous (with respect to $d^{\prime}$ ). Finally assume $x \in N$ with $T(x)=x$ and $\pi \mid U$ is a diffeomorphism of some neighborhood $U$ of $x$ onto a neighborhood $V$ of $\pi(x)$. Then $h_{d}(T)=k(\mu, T)=k\left(\mu^{\prime}, S\right)=h_{d^{\prime}}(S)$.

Proof. By Proposition 7 we need to show $k(\mu, T)=k\left(\mu^{\prime}, S\right)$ only. Choose $U_{1} \subset U$ a neighborhood of $x$ such that $T\left(U_{1}\right) \subset U$ and $\bar{U}_{1} \subset U$. Consider $\varepsilon>0$ with $B_{\varepsilon}(x) \subset U_{1}$. There are $a(\varepsilon)>0$ and $b(\varepsilon)>0$ such that

Then

$$
B_{a(\varepsilon)}(\pi(x)) \subset \pi B_{\varepsilon}(x) \subset B_{b(\varepsilon)}(\pi(x)) .
$$

$$
D_{n}(\pi(x), a(\varepsilon), S) \subset \pi D_{n}(x, \varepsilon, T) \subset D_{n}(\pi(x), b(\varepsilon), S) .
$$

Since $\pi \mid U$ is a diffeomorphism, $\mu$ and $\mu^{\prime}$ (positive) smooth measures, and $\bar{U}_{1}$ a compact subset of $U$, there are $C_{1}, C_{2}>0$ such that for any $E \subset U_{1}$ we have $C_{1} \mu^{\prime}(\pi(E)) \leqq \mu(E) \leqq C_{2} \mu^{\prime}(\pi(E))$. It follows that

$$
\begin{aligned}
\limsup _{n \rightarrow \infty}-\frac{1}{n} \log \mu^{\prime}\left(D_{n}(\pi(x), a(\varepsilon), S)\right) & \geqq \limsup _{n \rightarrow \infty}-\frac{1}{n} \log \mu\left(D_{n}(x, \varepsilon, T)\right) \\
& \geqq \limsup _{n \rightarrow \infty}-\frac{1}{n} \log \mu^{\prime}\left(D_{n}(\pi(x), b(\varepsilon), S)\right) .
\end{aligned}
$$

Now we can take $a(\varepsilon) \rightarrow 0$ and $b(\varepsilon) \rightarrow 0$ as $\varepsilon \rightarrow 0$; thus

$$
k\left(\mu^{\prime}, S\right) \geqq k(\mu, T) \geqq k\left(\mu^{\prime}, S\right) .
$$

COROLlARY 11. If $A$ is an endomorphism of a Lie group $G$ and $d$ is a right invariant metric, then

$$
h_{d}\left(R_{g} \circ A\right)=h_{d}(A)=h_{d}\left(d A \mid T_{e} G\right)
$$

Proof.

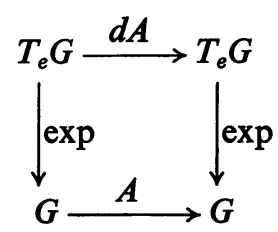

commutes and satisfies the hypotheses, where $\mu$ and $\mu^{\prime}$ are right Haar measures (by Example 8). See [16] for definitions.

\section{Linear maps.}

Proposition 12. Let $T: M^{m} \rightarrow M^{m}$ be a differentiable map of an m-dimensional Riemannian manifold. Then

$$
h_{d}(T) \leqq \max \left\{0, m \log \sup _{x \in M}\left\|d T \mid T_{x} M\right\|\right\} .
$$


Proof. $d$ of course is the metric determined by the Riemannian metric. Let $a=\sup _{x \in M}\left\|d T \mid T_{x} M\right\|$. If $a=\infty$, there is nothing to prove. Suppose $a<\infty$ and $K \subset M$ is compact. Let $f_{1}, \ldots, f_{r}: B_{3}(0) \subset R^{m} \rightarrow M$ be differentiable maps such that the $f_{i}\left(B_{1}(0)\right)$ cover $K$ and choose $A>0$ such that

$$
d\left(f_{i}(x), f_{i}(y)\right) \leqq A d(x, y)
$$

for all $x, y \in B_{2}(0)$ and all $1 \leqq i \leqq r$. If $a \leqq 1$, then $T$ never expands distances; in this case an $(1, \varepsilon)$-spanning set is an $(n, \varepsilon)$-spanning set and we get $h_{d}(T)=0$. Thus we may assume $a>1$.

For each $0<\delta \leqq 1$ let

$$
E(\delta)=\left\{\left(r_{1} \delta, \ldots, r_{m} \delta\right): r_{i} \in Z,\left|r_{i} \delta\right|<2\right\} .
$$

Then card $E(\delta) \leqq(5 / \delta)^{m}$ and there is a constant $B>0$ (depending on the metric $d$ used for $R^{m}$ ) such that for each $y \in B_{1}(0)$ there is an $x \in E(\delta)$ with $d(x, y) \leqq B \delta$. (For better constructions than this see [11].) Now $F(\delta)=\bigcup_{1 \leqq i \leqq r} f_{i} E(\delta)$ is clearly an ( $n, a^{n} A B \delta$ )-spanning set for $K$ (with respect to $T$ ) and card $F(\delta) \leqq(5 / \delta)^{m} r$. Hence, considering $\delta=\varepsilon / a^{n} A B$,

$$
r_{n}(\varepsilon, K) \leqq\left(5 a^{n} A B / \varepsilon\right)^{m} r=\left[(5 A B / \varepsilon)^{m} r\right] a^{n m}
$$

and so $\bar{r}_{T}(\varepsilon, K) \leqq m \log a$. Varying $\varepsilon$ and $K, h_{d}(T) \leqq m \log a$.

We recover a result due to Kouchnirenko [17]:

COROLlARY 13. If $T: M^{m} \rightarrow M^{m}$ is a differentiable map of a compact manifold and $\mu$ is a T-invariant Borel measure with $\mu(M)=1$, then

$$
h_{\mu}(T) \leqq \max \left\{0, m \log \sup _{x \in M}\left\|d T \mid T_{x} M\right\|\right\} .
$$

Proof. Use Goodwyn's theorem.

COROLlaRY 14. If $T: R^{m} \rightarrow R^{m}$ is a linear map, then

$$
h_{d}(T) \leqq \max \{0, n \log |\lambda|\}
$$

where $\lambda$ is an eigenvalue of $T$ with maximum absolute value.

Proof. By Propositions 12 and 4(i)

$$
\begin{aligned}
h_{d}(T)=\frac{1}{n} h_{d}\left(T^{n}\right) & \leqq \frac{1}{n} \max \left\{0, m \log \left\|T^{n}\right\|\right\} \\
& \leqq \max \left\{0, m \log \left\|T^{n}\right\|^{1 / n}\right\} .
\end{aligned}
$$

But $\left\|T^{n}\right\|^{1 / n} \rightarrow|\lambda|$ and $n \rightarrow \infty$ is a well-known theorem in analysis (see [18]).

THEOREM 15. If $T: R^{m} \rightarrow R^{m}$ is a linear map, then

$$
h_{d}(T)=\sum_{\left|\lambda_{i}\right|>1} \log \left|\lambda_{i}\right|
$$

where $\lambda_{1}, \ldots, \lambda_{m}$ are the eigenvalues of $T$. 
Proof. By linear algebra we can decompose $T=T_{1} \times \cdots \times T_{s}$ and $R^{m}=$ $E_{1} \times \cdots \times E_{s}$ where the $E_{j}$ are linear subspaces of $R^{m}$ with $T\left(E_{j}\right) \subset E_{j}, T_{j}=T \mid E_{j}$, and all the eigenvalues of $T_{f}$ have the same absolute value $\alpha_{j}$. Then

$$
\sum_{\left|\lambda_{i}\right|>1} \log \left|\lambda_{i}\right|=\sum_{\alpha_{j}>1} \operatorname{dim} E_{j} \log \alpha_{j} \text {. }
$$

By Proposition 4(ii) and Corollary 14 we need only to show that, for $\alpha_{j}>1$,

$$
h_{d}\left(T_{j}\right) \geqq \operatorname{dim} E_{j} \log \alpha_{j}=\log \left|\operatorname{det} T_{j}\right| \text {. }
$$

Letting $\mu$ be a Haar measure on $E_{j}$,

$$
\mu\left(D_{n}\left(0, \varepsilon, T_{j}\right)\right) \leqq \mu\left(T_{j}^{-n} B_{\varepsilon}(0)\right)=\mu\left(B_{\varepsilon}(0)\right) /\left|\operatorname{det} T_{j}\right|^{n} .
$$

Hence

$$
k\left(\mu, T_{\jmath}\right) \geqq \log \left|\operatorname{det} T_{j}\right| .
$$

By Proposition 7 and Example 8, $\mu$ is $T_{f}$-homogeneous and $h_{d}\left(T_{j}\right)=k\left(\mu, T_{j}\right)$. We are done.

COROLlaRY 16. If $A$ is an endomorphism of a Lie group $G$ and $d$ is a right invariant metric, then

$$
h_{d}(A)=\sum_{\left|\lambda_{t}\right|>1} \log \left|\lambda_{1}\right|
$$

where $\lambda_{1}, \ldots, \lambda_{m}$ are the eigenvalues of $d A \mid T_{e} G$.

Proof. Corollary 11 and Theorem 15.

REMARK. With Theorem 9 the above result gives the formula for $h_{\mu}\left(R_{g} \circ A\right)$ when $A$ is a surjective endomorphism of a compact Lie group $G$. For $G$ a torus this formula has a long history (see the introduction).

\section{Quotients.}

THEOREM 17. Let $(X, d),(Y, e)$ be compact metric spaces and $T: X \rightarrow X$, $S: Y \rightarrow Y, \pi: X \rightarrow Y$ (surjective) be continuous maps with $\pi \circ T=S \circ \pi$. Then

$$
h_{d}(T) \leqq h_{e}(S)+\sup _{y \in Y} h_{d}\left(T, \pi^{-1}(y)\right) .
$$

Proof. Clearly we may assume that $a=\sup _{y \in Y} h_{d}\left(T, \pi^{-1}(y)\right)<\infty$. Consider $\varepsilon>0$. Let $\alpha>0$ and for each $y \in Y$ choose $m(y)$ with

$$
a+\alpha \geqq h_{d}\left(T, \pi^{-1}(y)\right)+\alpha \geqq \frac{1}{m(y)} \log r_{m(y)}\left(\varepsilon, \pi^{-1}(y)\right) .
$$

Let $E_{y}$ be an $(m(y), \varepsilon)$-spanning set with the minimum number of members. Then $U_{y}=\bigcup_{z \in E_{y}} D_{m(y)}(z, 2 \varepsilon, T)$ is an open neighborhood of $\pi^{-1}(y)$.

Now

$$
\left(X \backslash U_{y}\right) \cap \bigcap_{y>0} \pi^{-1}\left(\overline{B_{y}(y)}\right)=\varnothing .
$$


By the finite intersection property for compact sets, there is a $W_{y}=B_{y}(y)$ for which $U_{y} \supset \pi^{-1}\left(W_{y}\right)$. Let $W_{y_{1}}, \ldots, W_{y_{r}}$ cover $Y$ and $\delta$ be a Lebesgue number for $Y$ for this open cover. Let $E_{n}$ be an $(n, \delta)$-spanning set for $Y$ (with respect to $S$ ) with the minimum number of elements. For $x \in E_{n}$ and $0 \leqq j<n$ pick $c_{j}(x)$ from among $y_{1}, \ldots, y_{r}$ such that

$$
\bar{B}_{\delta}\left(S^{j}(x)\right)=\left\{w: e\left(w, S^{\prime}(x)\right) \leqq \delta\right\} \subset W_{c_{j}(x)} .
$$

Define recursively $t_{0}(x)=0$ and $t_{s+1}(x)=t_{s}(x)+m\left(c_{\left(t_{s} x\right)}(x)\right)$ until one gets a $t_{q+1}(x) \geqq n$; set $q(x)=q$. For $x \in E_{n}$ and $z_{1} \in E_{c_{t_{1}(x)}(x)}, \ldots, z_{q} \in E_{c_{t_{q}(x)}(x)}$ define

$$
V\left(x ; z_{1}, \ldots, z_{q}\right)=\left\{u \in X: d\left(T^{t+t_{s}(x)}(u), T^{t}\left(z_{s}\right)\right)<2 \varepsilon\right.
$$

for all $0 \leqq t<m\left(c_{t_{s}(x)}(x)\right)$ and $\left.1 \leqq s \leqq q(x)\right\}$.

Then $\bigcup_{x, z_{1}, \ldots, z_{q(x)}} V\left(x ; z_{1}, \ldots, z_{q}\right)=X$ and $F \cap V\left(x ; z_{1}, \ldots, z_{q}\right)$ can have at most one element when $F$ is an $(n, 4 \varepsilon)$-separated set with respect to $T$.

For $x \in E_{n}$ the number of permissible $\left(z_{1}, \ldots, z_{q(x)}\right)$ is

$$
N_{x}=\prod_{s=0}^{q} r_{m\left(c_{t_{s}(x)}(x)\right)}\left(\varepsilon, \pi^{-1}\left(c_{t_{s}(x)}(x)\right)\right) \text {. }
$$

Let $M=\max \left\{m\left(y_{1}\right), \ldots, m\left(y_{r}\right)\right\}$. Then

$$
\begin{aligned}
\log N_{x} & \leqq \sum_{s=0}^{q(x)} \log r_{m\left(c_{t_{s}(x)}(x)\right)}\left(\varepsilon, \pi^{-1}\left(c_{t_{s}(x)}(x)\right)\right) \\
& \leqq(a+\alpha) \sum_{s=0}^{q} m\left(c_{t_{s}(x)}(x)\right) \leqq(a+\alpha)(n+M)
\end{aligned}
$$

and $N_{x} \leqq e^{(a+\alpha)(n+M)}$. By an observation we made above

$$
s_{n}(4 \varepsilon, X, T) \leqq \operatorname{card} E_{n} e^{(a+\alpha)(n+M)}
$$

and so $\bar{s}_{T}(4 \varepsilon, X) \leqq \bar{r}_{S}(\delta, Y)+a+\alpha \leqq h_{e}(S)+a+\alpha$. But $\alpha>0$ was arbitrary; let $\varepsilon \rightarrow 0$.

Corollary 18. Let $(X, d),(Y, e)$ be compact metric spaces and $T: X \rightarrow X$ and $\pi: X \rightarrow Y$ be continuous with $\pi \circ T=\pi$. Then

$$
h_{d}(T)=\sup _{y \in Y} h_{d}\left(T \mid \pi^{-1}(y)\right) .
$$

Proof. Theorem 17 gives an inequality in one direction since $h_{e}\left(\mathrm{id}_{Y}\right)=0$. The reverse inequality is obvious.

Remark. The above corollary answers a conjecture of [1]. R. Ellis and $\mathbf{H}$. Keynes helped to formulate the statement of Theorem 17. H. Keynes [15] contains Theorem 17 for the case of an isometric extension.

Let $(X, d),(G, d)$, and $(Y, d)$ be compact metric spaces. Assume there are continuous maps $\pi: X \rightarrow Y$ and $P: X \times G \rightarrow X$ (we write $P(x, g)=x g$ ) such that:

(1) $\pi$ is surjective, 
(2) $\pi^{-1}(\pi(x))=x G$, and

(3) $x g=x g^{\prime}$ only when $g=g^{\prime}$.

Suppose $T: X \rightarrow X, S: Y \rightarrow Y$, and $\tau: G \rightarrow G$ are continuous maps so that $S \circ \pi=\pi \circ T$ and $T(x g)=T(x) \tau(g)$. Then we say $T$ is a (right) $(G, \tau)$-extension of $S$. (Usually $G$ would be a group and $\tau$ an automorphism.)

THEOREM 19. In the above situation $h_{d}(T)=h_{e}(S)+h_{d}(\tau)$.

Proof. We first show that $h_{d}\left(T, \pi^{-1}(y)\right) \leqq h_{d}(\tau)$. Choose $z \in \pi^{-1}(y)$ and $\varepsilon>0$. As $(x, g) \rightarrow x g$ is uniformly continuous choose $\delta>0$ such that $d\left(x g, x g^{\prime}\right) \leqq \varepsilon$ whenever $d\left(g, g^{\prime}\right) \leqq \delta$. If $E_{n}(n, \delta)$-spans $G$ with respect to $\tau$, then $z E_{n}(n, \varepsilon)$-spans $\pi^{-1}(y)$ with respect to $T$. For if $d\left(\tau^{k}(g), \tau^{k}\left(g^{\prime}\right)\right) \leqq \delta$, then

$$
d\left(T^{k}(z g), T^{k}\left(z g^{\prime}\right)\right)=d\left(T^{k}(z) \tau^{k}(g), T^{k}(z) \tau^{k}\left(g^{\prime}\right)\right) \leqq \varepsilon .
$$

Thus $r_{n}\left(\varepsilon, \pi^{-1}(y), T\right) \leqq r_{n}(\delta, G, \tau)$ and we get $h_{d}\left(T, \pi^{-1}(y)\right) \leqq h_{d}(\tau)$. By Theorem 17, $h_{d}(T) \leqq h_{e}(S)+h_{d}(\tau)$.

Let $\varepsilon>0$. Choose $\delta>0$ so that

(i) $d(\pi(x), \pi(z)) \leqq \varepsilon$ when $d(x, z) \leqq \delta$ and

(ii) $d\left(x g, x g^{\prime}\right)>\delta$ when $x \in X$ and $d\left(g, g^{\prime}\right)>\varepsilon$.

By uniform continuity (i) holds for sufficiently small $\delta$. If (ii) did not hold for some small $\delta$, then we would have $x_{n} \in X$ and $g_{n}, g_{n}^{\prime} \in G$ with $d\left(g_{n}, g_{n}^{\prime}\right)>\varepsilon$ but $d\left(x_{n} g_{n}, x_{n} g_{n}^{\prime}\right) \rightarrow 0$. Choosing subsequences we may suppose $g_{n} \rightarrow g, g_{n}^{\prime} \rightarrow g^{\prime}$ and $x_{n} \rightarrow x$. Then $d\left(x g, x g^{\prime}\right)=0$ but $d\left(g, g^{\prime}\right) \geqq \varepsilon>0$; this contradicts condition (3) above.

Suppose $G_{n} \subset G$ is $(n, \varepsilon)$-separated (w.r.t. $\left.\tau\right)$ and $Y_{n} \subset Y$ is $(n, \varepsilon)$-separated (w.r.t. $S$ ). Choose $X_{n} \subset X$ with $\pi$ a bijection from $X_{n}$ to $Y_{n}$. We claim $X_{n} G_{n}$ is $(n, \delta)$-separated (w.r.t. $T)$. Consider $x g \neq x^{\prime} g^{\prime}$ from $X_{n} G_{n}$. If $x \neq x^{\prime}$, then $\pi(x) \neq \pi\left(x^{\prime}\right)$ are elements of $Y_{n}$ and so there is a $k$ for which $0 \leqq k<n$ and $e\left(\pi T^{k}(x g), \pi T^{k}\left(x^{\prime} g^{\prime}\right)\right)$ $=e\left(S^{k} \pi(x), S^{k} \pi\left(x^{\prime}\right)\right)>\varepsilon$. By (i) we have $d\left(T^{k}(x g), T^{k}\left(x^{\prime} g^{\prime}\right)\right)>\delta$. We need to consider now $x=x^{\prime}, g \neq g^{\prime}$. There is then a $0 \leqq k<n$ with $d\left(\tau^{k}(g), \tau^{k}\left(g^{\prime}\right)\right)>\varepsilon$. By (ii) we have

$$
d\left(T^{k}(x g), T^{k}\left(x g^{\prime}\right)\right)=d\left(T^{k}(x) \tau^{k}(g), T^{k}(x) \tau^{k}\left(g^{\prime}\right)\right)>\delta .
$$

Since $X_{n} G_{n}$ is $(n, \delta)$-separated,

$$
s_{n}(\delta, X, T) \geqq s_{n}(\varepsilon, Y, S) s_{n}(\varepsilon, G, \tau) .
$$

It follows that $h_{d}(T) \geqq h_{e}(S)+h_{d}(\tau)$ and the theorem is proved.

Let $G$ be a locally compact metrizable group with right invariant metric $d$ and right Haar measure $\mu$. Suppose $\Gamma$ is a uniform discrete subgroup of $G$, i.e. $\Gamma$ is discrete and $G / \Gamma$ is compact. Assume $A$ is an endomorphism of $G$ with $A(\Gamma) \subset \Gamma$ and $g \in G$; then $T(x)=g A(x)$ induces a map $S$ on $G / \Gamma$ by $S(x \Gamma)=g A(x) \Gamma$. We have $S \circ \pi=\pi \circ T$ where $\pi(x)=x \Gamma$. One can define a measure $\mu^{*}$ on $G / \Gamma$ by requiring $\mu^{*}(\pi(E))=\mu(E)$ whenever $E \subset G$ is a measurable set and $\pi$ is one-to-one on $E$ (this definition works because of the right invariance of $\mu$ and the discreteness of $\Gamma$ ). 
ThEOREM 20. $\mu^{*}$ is $S$-homogeneous and $k\left(\mu^{*}, S\right)=k(\mu, T)$.

Proof. We define the metric on $G / \Gamma$ by

$$
d(x \Gamma, y \Gamma)=\inf _{h \in \Gamma} d(x, y h) .
$$

(This is indeed a metric $[14$, p. 36].)

As $\Gamma$ is discrete, $N \cap \Gamma=\{e\}$ for some neighborhood $N$ of $e$. Consider $x \in G$. There is a $\delta>0$ such that $x^{-1} B_{4 \delta}(e) x \cap \Gamma=\{e\}$. We claim that if $y, z \in B_{\delta}(x)$ $=B_{\delta}(e) x$, then $d(y \Gamma, x \Gamma)=d(x, y)$. Clearly $d(y \Gamma, x \Gamma) \leqq d(y, z)$; suppose $d(y, z h)$ $\leqq d(y, z)<2 \delta$ for some $h \in \Gamma, h \neq e$. Then

$$
\begin{aligned}
d(x, x h) & \leqq d(x, y)+d(y, z h)+d(z h, x h) \\
& <\delta+2 \delta+\delta=4 \delta .
\end{aligned}
$$

Hence $x h \in B_{4 \delta}(e) x$ and $h \in x^{-1} B_{4 \delta}(e) x$, a contradiction.

If $d(x \Gamma, y \Gamma)<\delta$, then $d(x, y h)<\delta$ for some $h \in \Gamma$ and $y \Gamma=y h \Gamma \in \pi B_{\delta}(x)$. Hence $\pi \mid B_{\delta}(x)$ is an isometry of $B_{\delta}(x)$ onto $B_{\delta}(x \Gamma)$. The same $\delta$ works for $x h$; for if

$$
h^{\prime} \in(x h)^{-1} B_{4 \delta}(e)(x h) \cap \Gamma
$$

and $h^{\prime} \neq e$, then

$$
h h^{\prime} h^{-1} \in x^{-1} B_{4 \delta}(e) x \cap \Gamma
$$

and $h h^{\prime} h^{-1} \neq e$. Thus, for each $x \Gamma \in G / \Gamma$, there is a $\delta=\delta(x \Gamma)>0$ such that $\pi$ maps $B_{\delta}(x h)$ isometrically onto $B_{\delta}(x \Gamma)$ for each $h \in \Gamma$.

As $G / \Gamma$ is compact, let $B_{\delta\left(x_{1} \Gamma\right)}\left(x_{1} \Gamma\right), \ldots, B_{\delta\left(x_{r} \Gamma\right)}\left(x_{r} \Gamma\right)$ cover $G / \Gamma$ and let $\varepsilon$ be a Lebesgue number for this cover. Then, for every $y \in G, \pi$ maps $B_{\varepsilon}(y)$ isometrically onto $B_{\varepsilon}(y \Gamma)$.

Consider $\alpha>0$ so small that $\alpha<\varepsilon$ and $T B_{\alpha}(y) \subset B_{\varepsilon}(T(y))$ for all $y \in G$ (uniform continuity). We claim that

$$
\pi D_{n}(y, \alpha, T)=D_{n}(y \Gamma, \alpha, S) .
$$

This is clearly true for $n=0$. Suppose it is true for $n-1 \geqq 0$. If $z \in D_{n}(y, \alpha, T)$, then $d\left(T^{k}(z), T^{k}(y)\right)<\alpha<\varepsilon$ for $0 \leqq k<n$, and so $d\left(S^{k}(z \Gamma), S^{k}(y \Gamma)\right)=d\left(T^{k}(z) \Gamma, T^{k}(y) \Gamma\right)<\alpha$; hence $z \Gamma \in D_{n}(y \Gamma, \alpha, S)$ and $\pi D_{n}(y, \alpha, T) \subset D_{n}(y \Gamma, \alpha, S)$. Consider

$$
z \Gamma \in D_{n}(y \Gamma, \alpha, S) \subset D_{n-1}(y \Gamma, \alpha, S) .
$$

By inductive hypothesis, $z h \in D_{n-1}(y, \alpha, T)$ for some $h \in \Gamma$. We claim that $z h \in D_{n}(y, \alpha, T)$; otherwise $d\left(T^{n-2}(z h), T^{n-2}(y)\right)<\alpha$ but $d\left(T^{n-1}(z h), T^{n-1}(y)\right) \geqq \alpha$. In this case, $T^{n-1}(z h) \in B_{\varepsilon}\left(T^{n-1}(y)\right)$ (by the choice of $\alpha$ ) and, since $\pi$ is an isometry on $B_{\varepsilon}\left(T^{n-1}(y)\right)$,

$$
d\left(S^{n-1}(z \Gamma), S^{n-1}(y \Gamma)\right)=d\left(T^{n-1}(z h), T^{n-1}(y)\right) \geqq \alpha .
$$

This contradicts $z \Gamma \in D_{n}(y \Gamma, \alpha, S)$. Thus $z \Gamma \in \pi D_{n}(y, \alpha, T)$ and we have shown that $D_{n}(y \Gamma, \alpha, S)=\pi D_{n}(y, \alpha, T)$. 
We have $\mu^{*}\left(D_{n}(y \Gamma, \alpha, S)\right)=\mu\left(D_{n}(y, \alpha, T)\right)$. Now

$$
T(x)=g A(x)=\left(g A(x) g^{-1}\right) g=R_{g} \circ(\operatorname{Ad} g \circ A)(x)
$$

falls under Example 8 in $\S 2$. Hence $\mu$ is $T$-homogeneous. It follows that $\mu^{*}$ is $S$ homogeneous and $k\left(\mu^{*}, S\right)=k(\mu, T)$.

REMARK. For the above case it of course follows that $h_{d}(S)=h_{d}(T)$; this can be generalized to (uniform) isometric coverings without using any measures. The statement of Theorem 20 comes from a suggestion of W. Parry. In [19] Parry has a formula for $h_{\mu} \cdot(S)$ in the nilmanifold case. This is obtained here for $G$ any Lie group by Theorems 9 and 20 and Corollary 16.

5. Flows. In this section we verify a conjecture of [1].

Definition. A uniformly continuous flow on $(X, d)$ is a family of maps $\left\{\varphi_{t}: X \rightarrow X / t \geqq 0\right\}$ with $\varphi_{s+t}=\varphi_{s} \circ \varphi_{t}$ and such that for any $t_{0}>0$ and $\varepsilon>0$ there is a $\delta>0$ for which $d\left(\varphi_{t}(x), \varphi_{t}(y)\right)<\varepsilon$ whenever $0 \leqq t \leqq t_{0}$ and $d(x, y)<\delta$.

Proposition 21. In the above situation $h_{d}\left(\varphi_{t}\right)=t h_{d}\left(\varphi_{1}\right)$ for all $t>0$.

Proof. We show that $h_{d}\left(\varphi_{t}\right) \leqq(t / s) h_{d}\left(\varphi_{s}\right)$ for any $s, t>0$. Given $\varepsilon>0$ choose $\delta>0$ such that $d\left(\varphi_{r}(x), \varphi_{r}(y)\right)<\varepsilon$ for all $0 \leqq r \leqq s$ whenever $d(x, y)<\delta$. Consider $K$ compact. If $E$ is an $(n, \delta)$-spanning set for $K$ with respect to $\varphi_{s}$, then $E(m, \varepsilon)$-spans $K$ with respect to $\varphi_{t}$ whenever $m t \leqq n s$ (this is analogous to the proof of 4(i)). Hence

$$
\begin{aligned}
r_{m}\left(\varepsilon, K, \varphi_{t}\right) & \leqq r_{[m t i s]+1}\left(\delta, K, \varphi_{s}\right), \\
\bar{r}_{\varphi_{t}}(\varepsilon, K) & \leqq \bar{r}_{\varphi_{s}}(\delta, K) \lim _{m \rightarrow \infty} \sup \frac{1}{m}\left(\left[\frac{m t}{s}\right]+1\right)=\frac{t}{s} \bar{r}_{\varphi_{s}}(\delta, K) \\
& \leqq \frac{t}{s} h\left(\varphi_{s}, K\right) .
\end{aligned}
$$

Letting $\varepsilon$ and $K$ vary, $h_{d}\left(\varphi_{t}\right) \leqq(t / s) h_{d}\left(\varphi_{s}\right)$.

\section{REFERENCES}

1. R. L. Adler, A. G. Konheim and M. H. McAndrew, Topological entropy, Trans. Amer. Math. Soc. 114 (1965), 309-319. MR 30 \#5291.

2. R. Bowen, Periodic points and measures for Axiom A diffeomorphisms, Trans. Amer. Math. Soc. (to appear).

3. L. W. Goodwyn, Topological entropy bounds measure theoretic entropy, Proc. Amer. Math. Soc. 23 (1969), 679-688.

4. - The product theorem for topological entropy (to appear).

5. J. Munkres, Elementary differential topology, Ann. of Math. Studies, no. 54, Princeton Univ. Press, Princeton, N. J., 1963, pp. 23-24. MR 29 \#623.

6. W. Parry, Entropy and generators in ergodic theory, Math. Lecture Note Series, Benjamin, New York, 1969.

7. Ja. G. Sinaĭ, On the concept of entropy of dynamic system, Dokl. Akad. Nauk SSSR 124 (1959), 768-771. (Russian) MR 21 \#2036a.

8. V. A. Rohlin, Exact endomorphisms of a Lebesgue space, Izv. Akad. Nauk SSSR Ser. Mat. 25 (1961), 499-530; English transl., Amer. Math. Soc. Transl. (2) 39 (1964), 1-36. MR 26 \#1423. 
9. A. L. Genis, Metric properties of endomorphisms of an r-dimensional torus, Dokl. Akad. Nauk SSSR 138 (1961), 991-993 = Soviet Math. Dokl. 2 (1961), 750-752. MR 34 \#2766.

10. D. Z. Arov, Calculation of entropy for a class of group endomorphisms, Zap. Meh.-Mat. Fak. Har'kov. Gos. Univ. i Har'kov. Mat. Obšc. (4) 30 (1964), 48-69. (Russian) MR 35 \#4368.

11. K. Kryzewski and W. Schlenk, On invariant measures for expanding differentiable mappings, Studia Math. 33 (1969), 83-92.

12. K. Berg, Thesis, Minnesota.

13. - Convolution of invariant measures, maximum entropy (to appear).

14. D. Montgomery and L. Zippin, Topological transformation groups, Interscience, New York, 1955. MR 17, 383.

15. H. B. Keynes, Lifting topological entropy, Proc. Amer. Math. Soc. 24 (1970), 440-445.

16. C. Chevalley, Theory of Lie groups. I, Princeton Math. Series, vol. 8, Princeton Univ. Press, Princeton, N. J., 1946. MR 7, 412.

17. D. Anosov and Ja. G. Sinaǐ, Certain smooth ergodic systems, Uspehi Mat. Nauk 22 (1967), no. 5 (137), 107-172 = Russian Math. Surveys 22 (1967), no. 5, 103-167. MR 37 \#370.

18. L. Loomis, An introduction to abstract harmonic analysis, Van Nostrand, Princeton, N. J., 1953, p. 75. MR 14, 883.

19. W. Parry, Ergodic properties of affine transformations and flows on nilmanifolds, Amer. J. Math. 91 (1969), 757-771.

UNIVERSITY OF WARWICK, COVENTRY, ENGLAND 